\title{
Dental management in Williams-Beuren syndrome: Case report
}

\section{Postępowanie stomatologiczne w zespole Williamsa-Beurena - opis przypadku}

\author{
Arlete González-Sotelo ${ }^{A-F}$, María A. Monter-García ${ }^{A-F}$, Rosalía Contreras-Bulnes ${ }^{A-F}$ \\ Dental Research and Advanced Studies Center, School of Dentistry, Autonomous University of the State of Mexico, Mexico \\ A - research concept and design; B - collection and/or assembly of data; C - data analysis and interpretation; \\ $D$ - writing the article; $E$ - critical revision of the article; $F$ - final approval of article
}

Address for correspondence

Rosalía Contreras-Bulnes

E-mail: rcontrerasb@uaemex.mx

\section{Funding sources}

none declared

Conflict of interest

none declared

Received on December 14, 2016

Revised on January 19, 2017

Accepted on January 19, 2017

\begin{abstract}
Williams-Beuren syndrome is a multisystem disorder caused by microdeletion of chromosome №. 7 at the $7 q 11.23$ band. The physical phenotype includes typical facial dysmorphism with a flat nasal bridge, short upturned nose, periorbital puffiness, long philtrum and delicate chin. Common features include supravalvular aortic stenosis, mental retardation, learning disabilities, growth delay, infantile hypercalcemia, hyperacusis, feeding difficulties, scoliosis, strabismus and oral abnormalities.

The purpose of this paper was to describe the pediatric dentistry management and dental findings of a boy aged 9 years 3 months with Williams-Beuren syndrome, who had had a painful lower right first molar because of a dental abscess that was treated under local anesthesia and antibiotic prophylaxis. Consultation with the child's cardiologist revealed pulmonary stenosis, interatrial communication, ventricular septal defect and patent ductus arteriosus. When treating a patient with Williams-Beuren syndrome, the cardiovascular disease, mental retardation and behavior of the child must be considered. Preventive measures, education, caregiver training and frequent consultations with the pediatric dentist are essential for these patients.
\end{abstract}

Key words: Williams-Beuren syndrome, dental management

Słowa kluczowe: zespółWilliamsa-Beurena, postępowanie stomatologiczne

D0I

$10.17219 / \mathrm{dmp} / 68579$

Copyright

() 2017 by Wroclaw Medical University

and Polish Dental Society

This is an article distributed under the terms of the

Creative Commons Attribution Non-Commercial License

(http://creativecommons.org/licenses/by-nc-nd/4.0/) 
Williams-Beuren syndrome, was described by Dr. J. C. P. Williams ${ }^{1}$ and Dr. A. J. Beuren. ${ }^{2}$ Also known as Williams syndrome (Online Medelian Inheritance in Man [OMIM] number, 194050), it is a multisystem disorder caused by microdeletion of chromosome No. $7^{3,4}$ at the $7 q 11.23$ band $^{4-8}$ spanning 1.5 million to 1.8 million base pairs and containing 26 to 28 genes. Prevalence estimation is approximately 1 in 10,000 persons; ${ }^{9}$ however 1 per $20,000-50,000$ live births has been reported. ${ }^{3,6}$ Furthermore, the disorder has no sex, race or ethnic predilection. ${ }^{3,4}$

The physical phenotype includes typical facial dysmorphism (elfin-like face), ${ }^{10}$ young children are often described as cute or pixie-like, with a flat nasal bridge, short upturned nose, periorbital puffiness, long philtrum and delicate chin, whereas older patients have slightly coarse features, with full lips, a wide smile and full nasal tip. ${ }^{9}$ In addition, common features include supravalvular aortic stenosis, mental retardation, learning disabilities, growth delay, infantile hypercalcemia, ${ }^{4,8,10,11}$ hyperacusis, feeding difficulties, scoliosis and strabismus. ${ }^{6,8,9,11}$

Patients also show some abnormalities both in primary and permanent teeth: a high incidence of caries, malocclusions, enamel hypoplasia, supernumerary teeth, oligodontia, microdontia, taurodontism, pulp stones, excessive interdental spacing, shorts roots and aberrant tooth shape including peg-shaped teeth. ${ }^{3}$

Diagnosis of Williams-Beuren syndrome is based on recognition of the aforementioned characteristics and there is no single treatment. It involves a combination of medical monitoring, anticipatory guidance, direct therapies, pharmacotherapy, surgery and adaptive changes. None of the available treatments are curative. ${ }^{9}$

The aim of this paper was to report the treatment, oral and dental findings of a boy with Williams-Beuren syndrome and dental management of several months' evaluation.

\section{Case report}

A 9-year-3-month-old boy, previously diagnosed with Williams-Beuren syndrome at 4 years old (Fig. 1a), who had had a painful lower right first molar because of a dental abscess, presented to the Postgraduate Pediatric Dentistry Clinic (Toluca, México). Radiographic evaluation showed a periapical lesion (Fig. 2).

The patient was treated with amoxicillin $(50 \mathrm{mg} / \mathrm{kg})$ 60 min before the procedure as antibiotic prophylaxis against infective endocarditis. Drainage of the dental abscess was done. The antibiotic therapy continued for seven days (50 mg/ $\mathrm{kg} /$ day in three divided doses).

Consultation with the child's cardiologist revealed pulmonary stenosis, interatrial communication, ventricular septal defect and patent ductus arteriosus.

According to the parents, the patient has had heart disease, diminished intellectual capability and feeding diffi- culties since birth and he is bothered by loud noises (hyperacusis). Additionally, he has never attended a dental clinic before.

Medical history revealed a full-term infant (39 weeks) with low Apgar score (3/7) and $3 \mathrm{~kg}$ birth weight. Newborn resuscitation was required. The patient had had chickenpox at age 2, a thermal burn of the scalp had occurred at the age of 3 years, resulting in alopecia which involves the right temporal bone area crossing the anterior segment of the parietal bone to reach its left portion (Fig. 1b). Regarding feeding habits, the mother reported breastfeeding until 5 months, bottle feeding was withdrawn at the age of 1 and low dairy product consumption due to hypercalcemia.

Clinical examination showed short stature, dolichofacial type of face, hypertelorism, strabismus, wide depressed nose, thick lips, convex profile with apparent mandibular retrognathism, deficient chin and clinodactyly (Fig. 1a, c).

Intraoral examination of the mucosa, hard and soft palate, tonsils, tongue, and mouth floor revealed no pathologic findings. Oral hygiene was poor (O'Leary plaque control record [PCR] of 100\%) and moderate gingival inflammation was found around most of the teeth. Additionally, wide dental arches, anterior interdental spacing, tongue thrusting and malocclusion were observed (Fig. 3a, b). Dental examination showed mixed dentition stage, hypoplasia of the 2 right first permanent molars, absence of the lower right second bicuspid (as corroborated by X-ray evaluation) and coronal discoloration of the maxillary left central incisor due to pulp necrosis by a traumatic lesion 7 months previously. Extensive caries were in the first right permanent molars and primary molars.

The goals of the dental treatment was to eliminate the etiological factors of caries and gingivitis, to treat the lesions and to restore the function of the dentition, as well as to educate the parents on how to establish proper preventive measures.

Dental treatment included: composite resin fillings, pulpotomy and stainless steel crown of primary mandibular first molar, apexification in the permanent maxillary left central and extractions in the permanent and primary molars. Mandibular primary first left molar extraction was followed by the placement of a band and loop space maintainer (Fig. 3c, d). All of these procedures were carried out under local anesthesia and antibiotic prophylaxis.

Immediate preventive management consisted of teaching and reinforcing brushing technique, a complete prophylaxis and fluoridated varnish application as well as pit and fissure sealants in the permanent maxillary left premolars.

Follow-up examinations were scheduled every 3 months for a year, and showed healthy gingival tissue and no new caries, as well as eruption of premolars. The space maintainer was removed (Fig. 3e, f). It was therefore decided to start the orthodontic treatment since oral health had been successfully maintained for an extended period. 

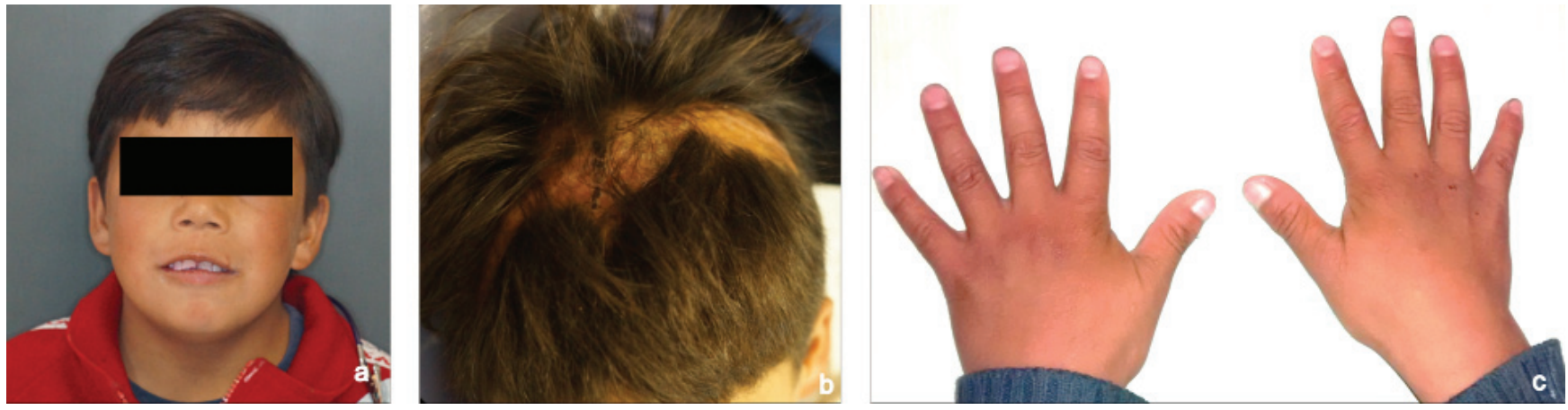

Fig. 1. Craniofacial views: a) facial dysmorphia; b) thermal burn of the scalp secondary to alopecia; c) view of hands (clinodactyly)

\section{Discussion}

Williams-Beuren syndrome is a very rare disorder and the extent of medical and developmental problems is highly variable. ${ }^{4,9}$ Cardiovascular and renal defects are the main problems we are confronted with, and moreover, the frequent occurrence of orofacial and dental abnormalities. ${ }^{3,10}$ In this case, the patient had pulmonary stenosis, interatrial communication, ventricular septal defect and patent ductus arteriosus, moderate intellectual disability, hyperacusis, hypercalcemia, strabismus, malocclusions and feeding difficulties. Stenosis of the medium and large arteries constitutes the prototypical cardiovascular abnormality of Williams-Beuren syndrome. ${ }^{9}$ Campos-Lara et al. ${ }^{3}$ reported some abnormalities both in primary and permanent teeth: malocclusion, enamel hypoplasia, oligodontia, pulp stones, microdontia, taurodontism, supernumerary teeth, excessive interdental spacing and short roots. Our patient presented enamel hypoplasia, malocclusion, hypodontia of the lower right second bi-

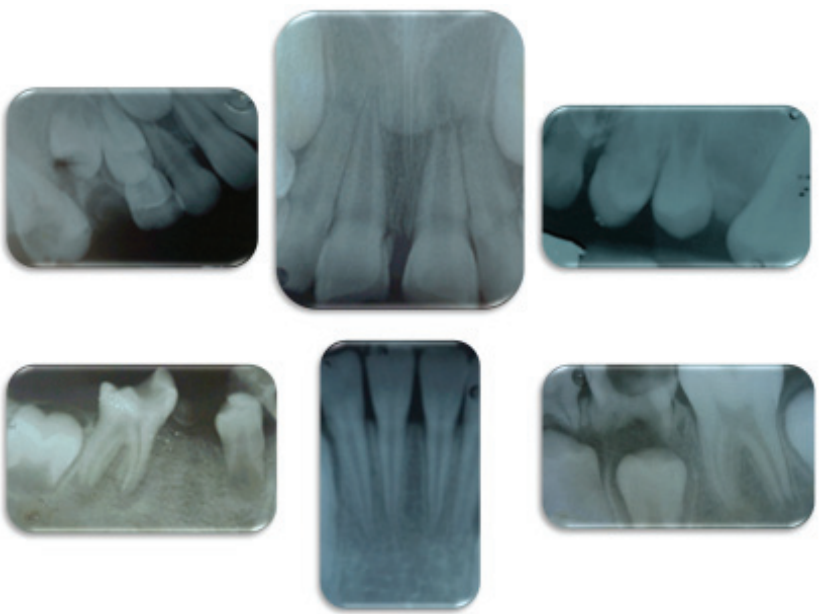

Fig. 2. Radiographic evaluation

cuspid and excessive interdental spacing. Consultation with a pediatric cardiologist is recommended for possible antibiotic prophylaxis, which in this case was required. However, Campos-Lara et al. ${ }^{3}$ described a case with
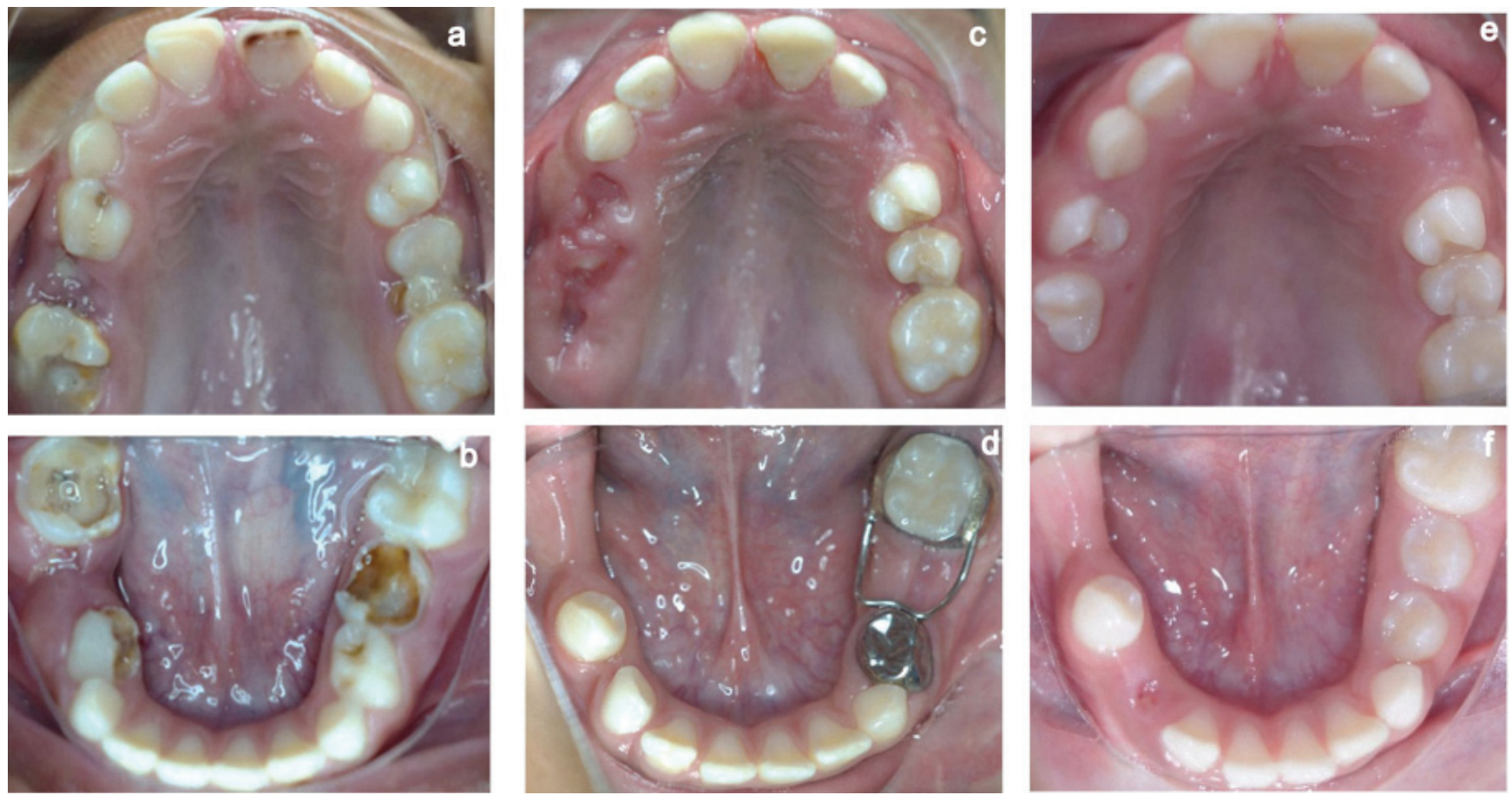

Fig. 3. Intraoral views: a, b) preoperative photographs; c, d) postoperative photographs; e, f) 1 year later, healthy gingival tissue and no new caries were observed 
Williams-Beuren syndrome presenting aortic stenosis and didn't report antibiotic prophylaxis. Moskovitz et al. ${ }^{4}$ reported 3 cases of Williams-Beuren syndrome with multiple decayed teeth, the patients had uncooperative behavior, the treatment was performed under sedation, and antibiotic prophylaxis was administrated in 2 cases.

Our patient, on the other hand, had cooperative behavior. The entire dental treatment was performed on an outpatient basis, which prevented the child from undergoing general anesthesia. Establishing a relationship with the patient, the use of communication techniques and positive reinforcement enabled the development of the child's positive attitude towards oral health, resulting in an improvement of oral hygiene and, therefore, maintaining oral health.

Treating a patient with Williams-Beuren syndrome should take into account cardiovascular disease, mental retardation and the behavior of the child. Preventive measures, education, caregiver training and frequent consultations with a pediatric dentist are essential for patients with special health care needs.

\section{References}

1. Williams JCP, Barrat-Bayers BG, Lowe JB. Supravalvular aortic stenosis. Circulation. 1961;21:1311-1318.

2. Beuren AJ, Apitz J, Harmjanz D. Supravalvular aortic stenosis in association with mental retardarion and certain facial appereance. Circulation. 1962;26:1235-1240.

3. Campos-Lara P, Santos-Díaz AM, Ruiz-Rodríguez MS. Orofacial finding and dental management of Williams-Beuren syndrome. J Clin Pediatr Dent. 2012, 36: 401-404.

4. Moskovitz M, Brener D, Faibis S, Peretz B. Medical considerations in dental treatment of children with Williams syndrome. Oral Surg Oral Med Oral Pathol Oral Radiol Endod. 2005;99:573-580.

5. Borg I, Delhanty JD, Baraitser M. Detection of hemizygosity at the elastin locus by FISH analysis as diagnostic test in both classical and typical cases of Williams syndrome. J Med Genet. 1995;32:692-696.

6. Damasceno ML, Cristine AF, Marcon RM, Barros Filho TE. Prevalence of scoliosis in Williams-Beuren syndrome patients treated at a regional reference center. Clinics. 2014;69:452-456.

7. Ueda K, Yamada J, Takemoto O, Okamoto N. Eight patients with Williams syndrome and craniosynostosis. Eur J Med Genet. 2015;58:355-357.

8. Sammour Z. Congenital genitourinary abnormalities in children with Williams-Beuren syndrome. J Pediatr Urol. 2014;10:804-809.

9. Pober BR. Williams-Beuren sydrome. N EnglMed. 2010;362:239-252.

10. Tordjam S. Presence of autism hyperserotonemia, and severe expressive language impairment in Williams-Beuren syndrome. Mol Autism. 2013;4:29.

11. Kirchner RM, Martens MA, Andridge RR. Adaptive behavior and development of infants and toddlers with Williams Syndrome. Front Psychol. 2016;7:598. 